\title{
ZAHA HADID: FLUIDEZ DE MOVIMIENTO
}

\author{
ZAHA HADID: FLOW MOVEMENT
}

\section{MAURICIO CABAS GARCÍA}

Arquitecto. Especialista en diseño arquitectónico de la Universidad Autónoma del Caribe. Docente de la Corporación Universidad de la Costa (CUC). mcabas1@cuc.edu.co

\section{RESUMEN:}

El objetivo de esta reflexión es describir el concepto arquitectónico de Zaha Hadid y su manera de crear o determinar el espacio sin aceptar formas predeterminadas, sino creando sus propias formas arquitectónicas; esto se conoce como arquitectura de determinación formal espacial, que no recurre a ninguna premisa histórica u objetiva, y el espacio nace con la evolución o el desarrollo de una idea de la forma arquitectónica propia. Este artículo analiza el manejo del espacio arquitectónico y cómo esta arquitecta crea una espacialidad única basada en formas curvas y fluidas, con alto grado de movimiento y levedad, así como un perfecto trabajo con la luz.

\section{PALABRAS CLAVE:}

Zaha Hadid, espacio arquitectónico, fluidez, movimiento, urbatectura, topografía, deconstructivismo, geometría, espacialidad líquida, luz

\section{ABSTRACT:}

Zaha Hadid is an architect who seeks to create or determine the space, she does not accept default, and creates her own architectural forms, this is known as spatial architecture formal determination, does not resort to any historical or factual premise and the space is born or created with the evolution or development of an idea of the architectural form itself. This article discusses the use of architectural space and how this architect creates a unique space-based curves and flowing shapes with a high degree of movement and lightness, and a perfect work to light.

\section{KEYWORDS:}

Zaha Hadid, architectural space, fluency, movement, urbatechture, topography, deconstrutivism, geometry, liquid space, light 


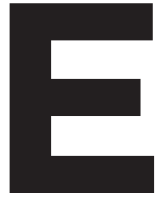

I lenguaje arquitectónico de Zaha Hadid se caracteriza por la fortaleza de sus líneas y curvas, en el cual el espacio arquitectónico se comporta como un fluido, demostrando gran expresividad, con un dinamismo extremadamente marcado y basado en la topografía del terreno. Es un espacio que busca la estimulación de las personas. "Como arquitectos debemos respetar las costumbres de la gente, pero al mismo tiempo proyectar formas de vida que resulten estimulantes". (Hadid, 2001).

Evidentemente la arquitectura de Zaha Hadid se relaciona en gran medida con su contexto, produciendo una construcción e identificación de lugar única, hasta el punto de mezclarse lo artificial y lo natural en una sola obra. "Una nueva relación entre el objeto arquitectónico y su contexto parece dar lugar a un "paisaje informático", en el que, gracias a las más modernas manipulaciones de la Tecnología Informática, la artificiosidad de la arquitectura viene a coincidir con la verdadera construcción del lugar. La arquitectura relacionada con las fuerzas esenciales, cósmicas y naturales del lugar se transforma en una "solidificación natural y topográfica" de la tierra, hasta convertirse en una forma de Land Art". (Barborsky, 2001). Cabe resaltar que en sus inicios el concepto arquitectónico de Zaha Hadid fue influenciado por el movimiento vanguardista ruso, pero que poco a poco fue ideando una manera diferente de ver la arquitectura, más hacia lo sensible, más hacia lo sorprendente.

“La fase más temprana del movimiento moderno era inspiradora y la vanguardia rusa lo era aún más. Sin embargo, mi trabajo actual ya no está marcado por tales influencias. Cuando estudiaba quizá era estimulante, pero ahora me resulta más inspirador viajar, llegar a un lugar totalmente nuevo o descubrir un paisaje. También la música puede ser una fuente de inspiración. Ya no se trata de una inspiración directa, sino de crear algo a partir de las obras propias. Uno va desarrollando un lenguaje arquitectónico y cuando al hacerlo realiza descubrimientos poco comunes, se llega también a un lenguaje singular. La arquitectura me sigue pareciendo algo muy emocionante, pero hay otras cosas que también lo son". (Hadid, 2006).

\section{EL ESPACIO ARQUITECTÓNICO DE ZAHA HADID}

El espacio arquitectónico creado por Zaha Hadid se ge-nera por medio de un proceso artístico en el cual la forma del paisaje y la geometría tienen un papel importantísimo, brindándole a la obra arquitectónica su carácter de articulador entre lo natural y lo artificial. Podríamos hablar de "urbatectura": no se sabe el límite exacto entre la obra arquitectónica y el paisaje o el espacio público.

Clara evidencia de esto es el proyecto concebido en Alemania a las orillas del río Rhein, el Pabellón Landgartenschau, en el cual se aprecia la relación entre la naturaleza y la obra construida. El pabellón se convierte en punto de recreo y de descanso dentro de un jardín y consolida su topografía. Barborsky (2001) expresa que específicamente la composición de este edificio deriva de los principios ordenadores de los recorridos peatonales del jardín, tres de los cuales, entrelazándose, configuran la planta del edificio.

El primer corredor flanquea el lado sur del Pabellón; el segundo, siguiendo la suave pendiente de una rampa lleva hasta la cubierta, y el tercero, describiendo una doble y profunda curva, lo atraviesa por el lado más corto. De este entrelazado de directrices, el Pabellón hace derivar la distribución de sus espacios interiores, parcialmente conectados entre sí. Una sala expositiva y una cafetería están situadas a lo largo de los perfiles del edificio, con el fin de que gocen de iluminación natural, ofreciendo, al mismo tiempo, la vista de la naturaleza circundante. El núcleo del edificio está, en cambio, ocupado por oficinas y salas secundarias, mientras que un corredor peatonal lleva a la terraza y a la cubierta del pequeño centro de investigaciones ambientales, situado al norte de la sala expositiva y parcialmente enterrado, garantizando así la continuidad peatonal entre los recorridos del jardín y la fruición del edificio. Las latentes y ocultas sugerencias del lugar son hábilmente transformadas por Hadid en formas arquitectónicas y distribuciones planimétricas capaces de conformarse en un nuevo paisaje artificial. (Barborsky, 2001).

El deconstructivismo de Zaha Hadid surge como reacción o respuesta al historicismo que se dio en la arquitectura en los años setenta y ochenta; de igual manera, es decir adiós a la arquitectura modernista que producía bloques fortificados. De allí que la arquitectura y el espacio que crea Hadid busca un lenguaje permeable y social, así como un espacio fluido y fragmentado, y una volumetría que estimule los sentidos y abierta.

El espacio arquitectónico concebido por Zaha Hadid cuenta con unos principios elementales de configuración, como lo son la geometría, el movimiento y una organización espacial muy racional, aunque no lo parezca. La misma Hadid (2005) comenta que con el movimiento y la espacialidad trata de crear un espacio que flota o gravita. Hay que entender muy bien el concepto de un espacio que gravita; la interpretación sería que el espacio sea liviano, un espacio basado en la levedad, en el dinamismo de los recorridos, en la generación de sensaciones deliciosas y sensuales, líneas y curvas que insinúan direcciones y expansiones.

Zaha Hadid plantea un proceso de diseño teniendo en cuenta orientaciones ya sean verticales u horizontales. Lo vertical implica utilizar la forma del edificio o la silueta del mismo teniendo como fondo o background el cielo, y no solo en lo volumétrico sino en también en el espacio interior. Por el contrario, lo horizontal implica la utilización y manipulación del suelo y el terreno como elemento compositivo de la arquitectura y el espacio, para de esa forma generar una topografía 


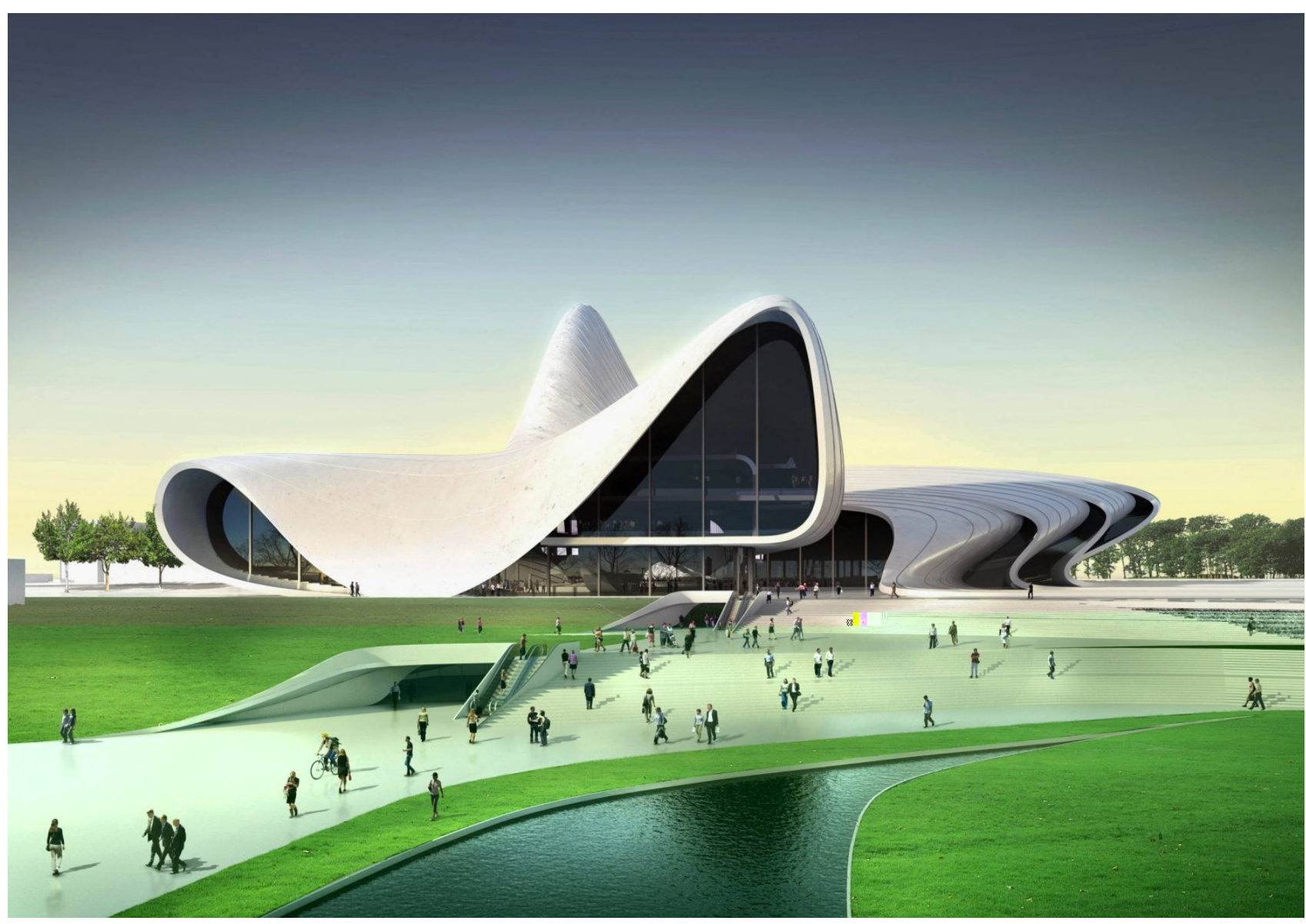

Imagen 1. Hayda Rend, Zaha Hadid Architects.

artificial para crear nuevas situaciones y tensiones urbanas.

" Toda esa idea del suelo y de la manipulación del suelo tiene su origen en mis primeros proyectos. Yo diría que se remonta a la Residencia para el Primer Ministro Irlandés porque aquel proyecto ya implicaba esa idea que tiene que ver con el paisaje artificial, y también a los estudios para los extensos campos de Vitra; y más tarde cuando hicimos el V\&A, donde la idea se desarrollaba en un conjunto interior escalonado, como si un paisaje pastoral comenzase realmente a ser succionado hacia el interior." (Hadid, 2001).

De igual manera, Hadid maneja el concepto del pixelado y de la agregación, lo cual tiene que ver con las tensiones y fuerzas de atracción de las distintas formas y volúmenes con el ejercicio de la manipulación del suelo. Se está estableciendo un juego de flujos y fuerzas y sus influencias en puntos externos y extremos.

La arquitectura de Zaha Hadid se enrolla y se desborda, se mueve sobre sí misma, generando volúmenes que se friccionan y se funden, así como ella misma comenta, se convierten en paisajes solapados.
Como los trazados en delta para Roma, que se desbordan horizontalmente; y por otra parte, paralelamente, trabajamos en proyectos donde esos campos se enrollan y se mueven unos sobre otros de manera más ajustada, y se convierten en paisajes solapados, como en las colecciones Reales de Madrid o en esos cuerpos enrollados y compactados del Reina Sofía. Poco después, en los proyectos de Graz y Montreal, esas fuerzas fluidas se expresan con mayor claridad y se colocan de modo que crean una fuerte tensión con las estructuras tectónicas dentro de las cuales están moldeadas o encarceladas. (Hadid, 2001).

La idea del pixelado genera un sistema estándar para generar la máxima fluidez, lo cual conduce a la idea de la repetición. La idea es involucrar el exterior al espacio interior, succionar el urbanismo del entorno y el paisaje hacia el interior del edificio, produciendo densidades y una espacialidad casi líquida.

Puede que una haga referencia a una escala más condensada, probablemente inclinada hacia una dimensión vertical, mientras que la otra lleva implícita la noción de un campo horizontalmente expansivo, un campo que empieza a conver- 


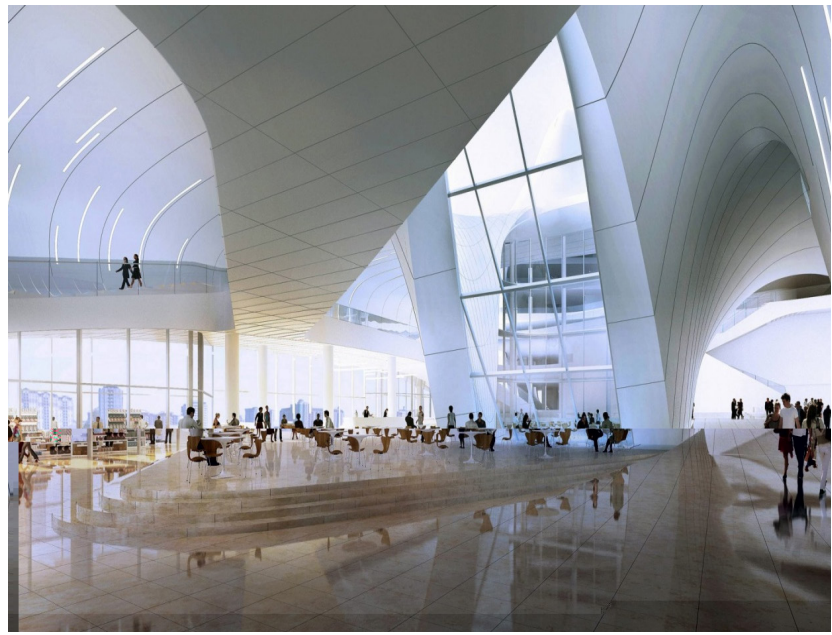

Imagen 2. Hayda Rend, Zaha Hadid Architects

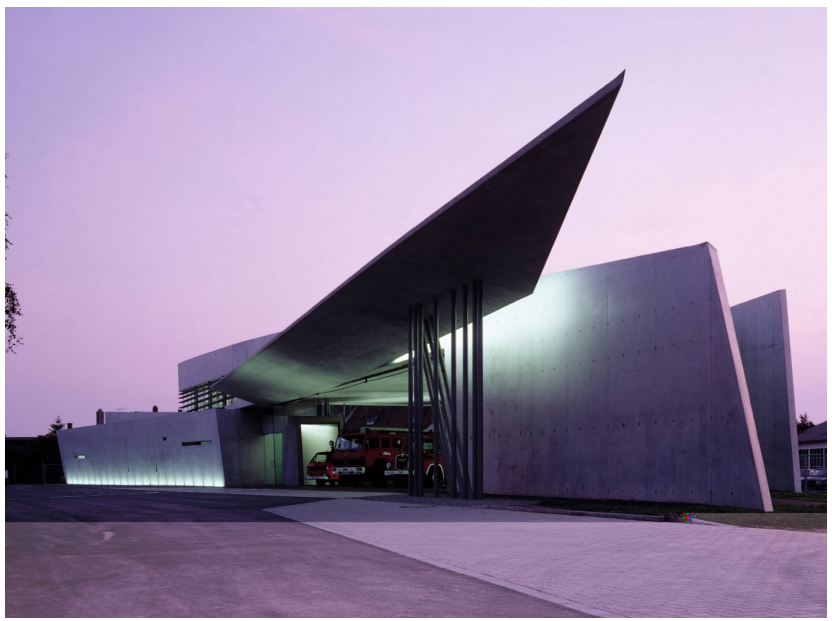

Imagen 3. Estación de Bomberos. Fotografía de Cristian Richter.



Imagen 4. Irish Rend, Zaha Hadid Architects. tirse en algo parecido a una organización urbana desplegada sobre un gran terreno, aunque todavía condensada, implosionada e intensificada. (Hadid, 2001).

El tema fundamental en la concepción espacial por parte de Zaha Hadid continúa siendo entonces la apropiación del terreno y su topografía, así como la complejidad de la geométrica interior mediante una condensación de orientaciones y ejes relacionados con su entorno. Sus proyectos hacen emerger recorridos urbanos que en muchos casos permanecen ocultos o en silencio por los transeúntes y que solo son descubiertos cuando se experimenta el espacio y se implanta su obra. “La arquitectura facilita todo esto tratando por igual los espacios exteriores y los interiores; es decir, que los espacios interiores tienen la máxima luz natural... nuestros proyectos se consideran factores de transformación". (Hadid, 2001).

El caso del proyecto realizado en Cincinnati, el centro de Arte Contemporáneo Rosenthal, se consideró como un elemento de la estructura del espacio público, como un acto público en sí mismo. Se convierte en una obra de arte como tal. Y su diseño parte del estudio del contexto y lo formal resulta de ese estudio. Pero existe algo muy marcado y es la forma de los recorridos interiores que plantean un concepto casi laberintico de la composición espacial, permitiendo a la persona ir descubriendo vistas diferentes y fugaces de los espacios que tiene por delante y que tiene que atravesar. Hadid (2001) expresa su gusto por el uso del hormigón, ya que con este, además de realizar la estructura portante, puede ejecutar el resto del edificio. Esa es la razón primordial por la cual escoge el hormigón para realizar sus proyectos. Continua Hadid (2001) explicando que el proceso sigue con los detalles de los acristalamientos, y en ese momento introduce algo de color. Aunque está abierta a utilizar otro tipo de material para utilizarlo en el cuerpo del edificio. La arquitectura de Zaha Hadid se caracteriza desde el punto de vista estético, y lo que además le da un carácter escultural es el uso del hormigón a la vista, ya que con este material logra solucionar el componente estructural y le da pureza a la obra. De igual manera, resalta el concepto de levedad, lo más pesado puede ser levantado y flotar.

El Centro de Arte Contemporáneo de Roma es un proyecto más abierto, debido al entorno en cual está implantado, pero de igual manera se logran apreciar los recorridos intrincados que se relacionan siempre con el exterior, permitiendo la entrada de luz natural. "En Roma el lugar realmente se convierte en parte de la ciudad. Ya no está fortificado; se puede caminar por él y recorrer una explanada pública con independencia de que se vayan a visitar o no las galerías". (Hadid, 2001).

Es un proyecto de muchos espacios abiertos, interesante para recorrer, y su organización espacial no es la típica de los museos normales, en los cuales se llega a un lugar del cual se accede a las distintas galerías; por el contrario, se puede acceder a todas las galerías sin necesidad de un lobby de reparto. 
En realidad hay muchos espacios abiertos en la planta baja del proyecto de Roma. No toda está ocupada. El emplazamiento de Roma es muy grande y está estructurado por campos de fuerzas y por trayectorias que atraviesan el solar. Siguiendo estas líneas de fuerza, rellenamos o "irrigamos" el solar con muros continuos. Cuando estos muros se cortan, crean y distribuyen tanto el espacio interior como el exterior. Así se crea mucho espacio abierto y mucho espacio intermedio. Eso ofrece al visitante múltiples opciones con respecto a su itinerario, que puede incluir el centro de arquitectura, la biblioteca o las galerías; por eso se trata realmente de un campus de las artes en contraposición al edificio como objeto único. Podemos elegir a qué sector del campus vamos a ir, e incluso cuando nos concentramos en el sector principal, que es el edificio de las galerías, de hecho podemos hacer un recorrido distinto cada vez que volvemos. (Hadid, 2001).

Esto nos lleva a la idea de recorridos con dinamismo y progresión espacial muy distinta a los recorridos lineales, como si estuviéramos hablando de distintos circuitos en un mismo espacio.

Otro proyecto extremadamente interesante de Zaha Hadid es el realizado en Dusseldorf (Alemania), el Centro de Arte Multimedia, debido a que en este caso también se manipula el suelo como una topografía artificial, mediante terrazas, multiplicando la superficie y estratificando. Con movimientos suaves a partir del nivel de la calle tanto hacia arriba como hacia abajo, y por encima de todo esto se encuentran bloques de oficinas cada uno sostenido por un sistema de columnas que asemejan un paisaje boscoso. Como Hadid (2001) explica, que los conceptos que dieron origen al proyecto diseñado y construido en Dusseldorf fueron la manipulación del terreno y la articulación de estructura como forma del paisaje, conceptos manejados también en el proyecto de la Opera en la Bahía de Cardiff.

El espacio arquitectónico en la Ópera de la Bahía de Cardiff ofrece un enorme espacio como plaza pública y como complemento de la plaza ovalada que existía con anterioridad. Pero esa plaza sube una pendiente para generar recorridos hacia el interior; de nuevo se aprecia lo que se comentó anteriormente, la urbatectura, y la luz se convierte en un personaje principal, ya que todo el espacio se abre para permitir su paso a los niveles inferiores. Así mismo, en planta se aprecia la fragmentación 0 , si se puede decir, una organización fragmentada que con recorridos de gran fluidez que responden al entorno. Hadid pretende cambiar el concepto general y predominante de estas tipologías, que generalmente son grandes volúmenes y masas sólidas en las cuales se encuentran las salas de conciertos.

Todo el suelo se infla formando una burbuja y ofrece este enorme espacio como explanada pública y como ampliación de plaza ovalada que está delante del edificio. Pero la plaza se bifurca en sección y se extiende también por la parte superior de la burbuja subiendo la pendiente. Luego la burbuja se corta

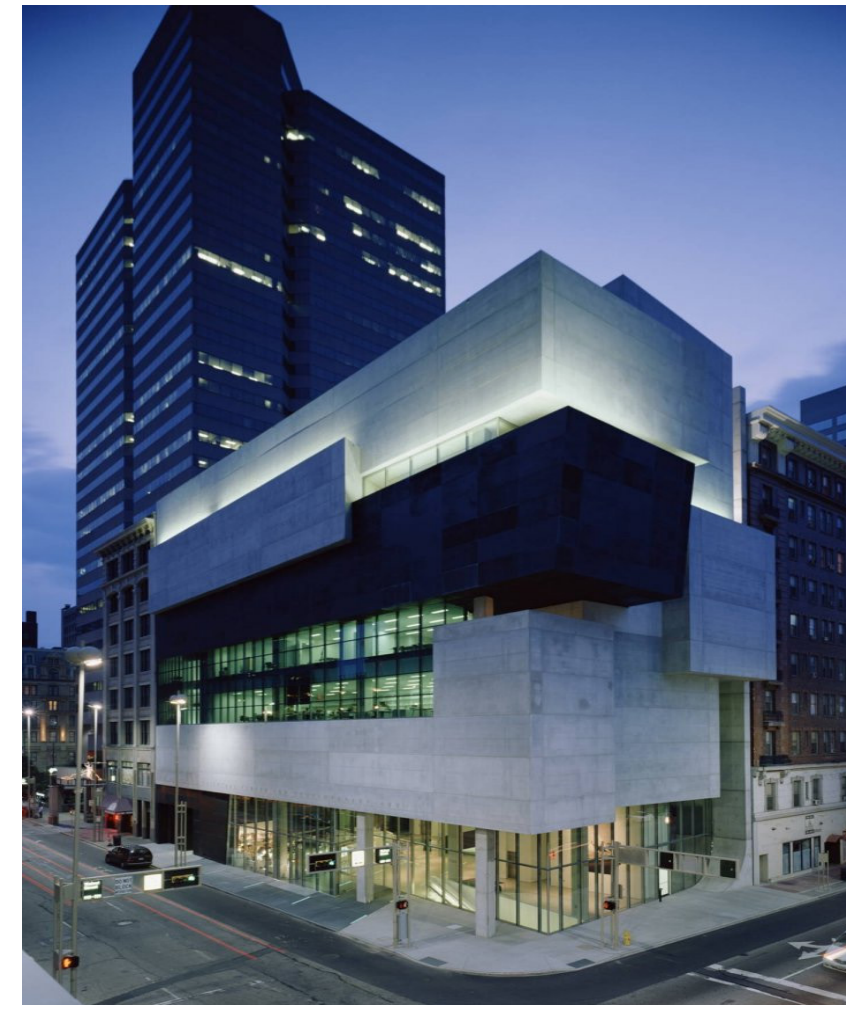

Imagen 5. Centro Rosenthal. Fotografia de Roland Halbe..

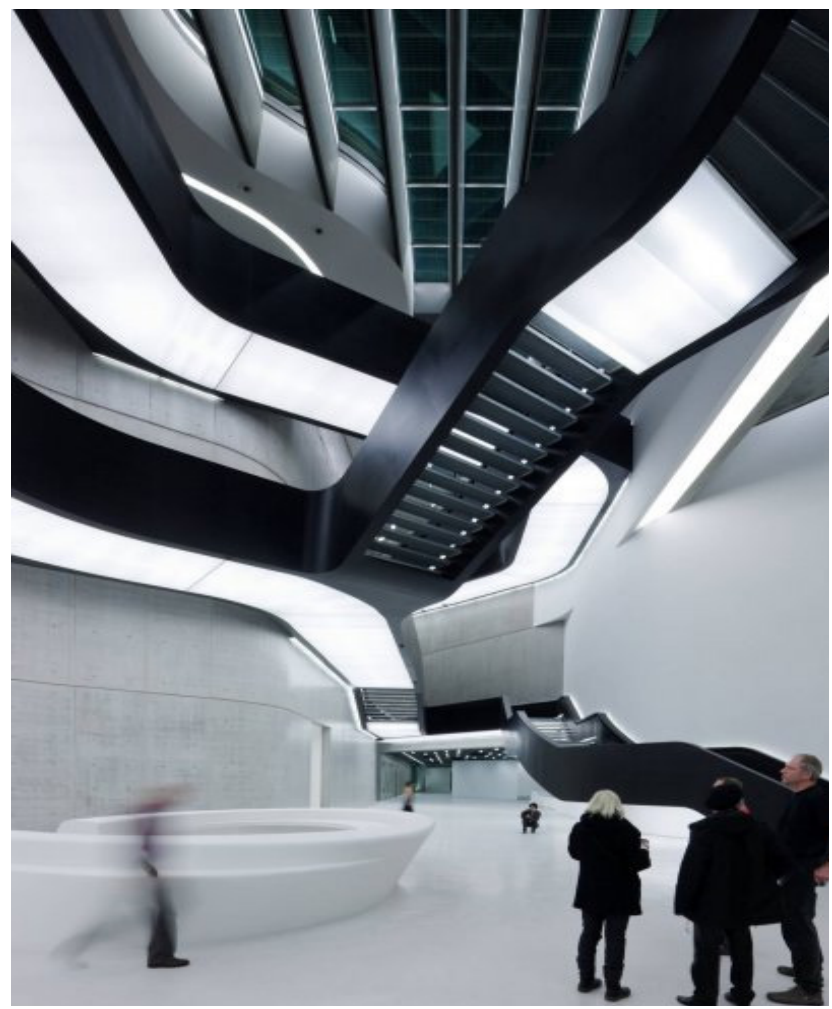

Imagen 6. Maxxi. Fotografia de Helene Binet. 


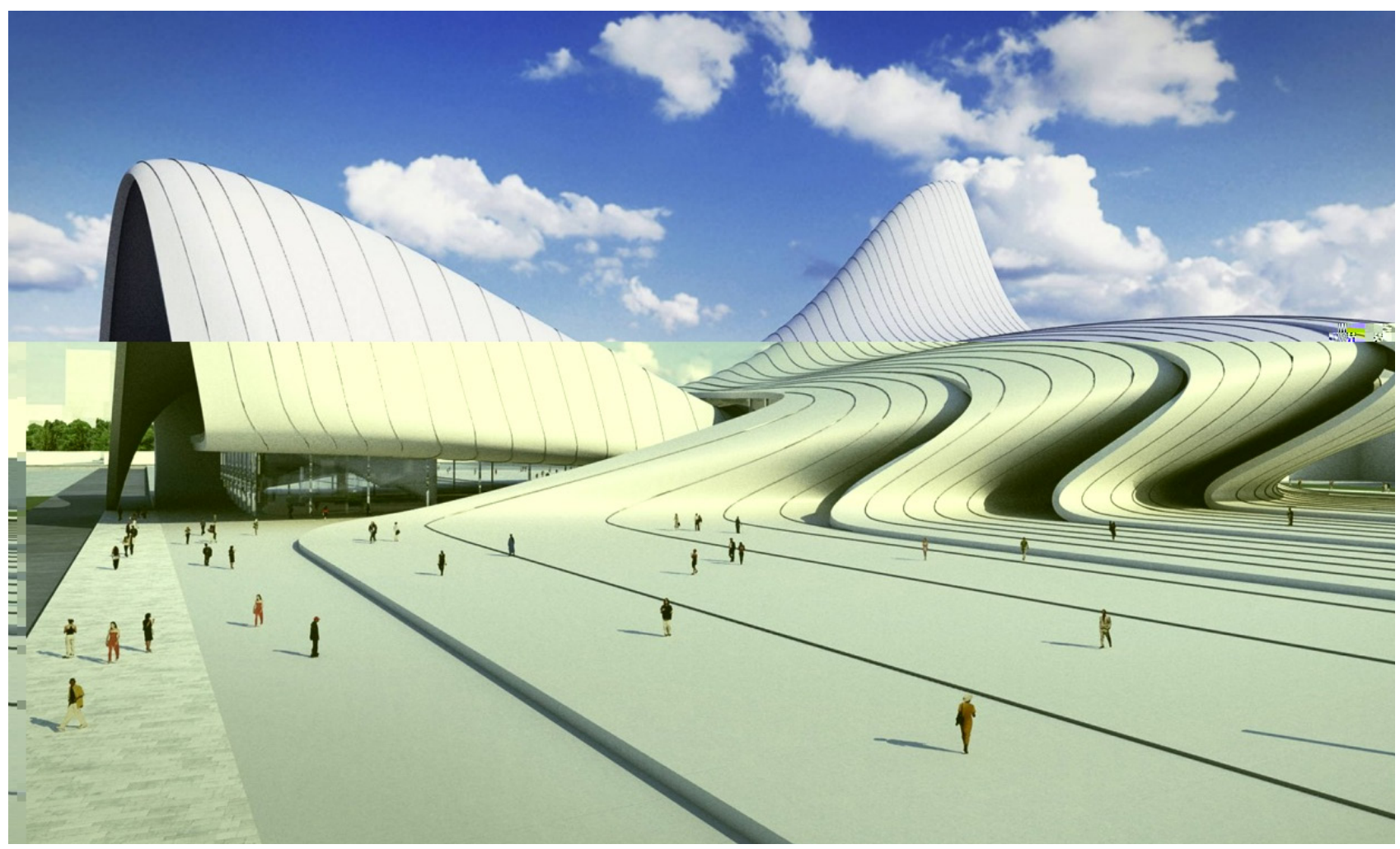

y se descorteza para hacer llegar la luz hasta el espacio que está debajo. Estas manipulaciones participan conjuntamente en el esfuerzo global por reconfigurar la tipología de los teatros de ópera... Lo que es interesante es la fragmentación de la planta, que con el tiempo entró en relación con los flujos urbanos y se hizo más fluida. Comenzamos a romper algunos obstáculos de la planta. Finalmente, los flujos y las piezas fragmentarias llegaron a ser una sola cosa en lugar de dos independientes. La fragmentación y el flujo se funden en la idea de campo. (Hadid, 2001)

Para Hadid (2001), el entorno urbano es un aspecto de suma importancia en sus diseños, es crucial y decisivo. De ahí que el Science Center, ubicado en la ciudad de Wolsburg, en Alemania, fue pensado para que su volumen no impida la relación visual entre los peatones y el resto de la ciudad; todo lo contrario, se hizo para buscar y amplificar dicha relación, generando un espacio público urbano debajo del volumen de la edificación. Durante el día, el interior del sitio es accesible para todas las personas, contando con relaciones directas con la estación de trenes cercana y los proyectos circundantes como la ciudad del automóvil. En la noche, el espacio urbano debajo del edificio se convierte en una plaza abierta con mucha vida social.

\section{CONCLUSIONES}

Zaha Hadid ha creado una arquitectura y una espacialidad que se mueve, que se curva, que se transforma bajo la influencia del sitio donde se implanta y del programa arquitectónico. Su arquitectura está pensada para ser disfrutada y recorrida tanto físicamente como con la mirada. Además de lo variable de la volumetría y la gran riqueza formal en la obra de Zaha Hadid, la organización espacial en planta sigue siendo crucial, pero no es esta la que produce el proyecto mediante su proyección o extrusión piso por piso. Todo lo contrario, la espacialidad en dos dimensiones va cambiando piso a piso en una forma muy flexible y muy lógica basada nuevamente en la geometría.

Hadid pretende siempre experimentar con nuevas organizaciones y composiciones planimétricas y geométricas, para de ese modo provocar distintas experiencias y acontecimientos espaciales poco comunes. Puede llegar a pensarse que los proyectos de Zaha Hadid son meramente concebidas como una escultura, pero eso es completamente alejado de la realidad. El aspecto social de la arquitectura y, por consiguiente, del espacio arquitectónico es para Hadid de gran importancia; todas las relaciones y tensiones urbanas son muy tenidas en cuenta en los planteamientos generales de cada uno de los proyectos que se le presentan. 
Para Zaha Hadid no es primordial el paradigma de la complejidad organizacional con la que los arquitectos suelen encarar y empezar a dibujar sus proyectos; aunque reconoce su importancia, en sus trabajos se logra observar la gran importancia que le da a las relaciones con el paisaje, la infraestructura, la biología y lo social. Y que en cierta manera le sirven como medio para explorar la forma. Zaha Hadid es reconocida por sus edificios espectaculares, en los cuales las curvas son los protagonistas principales ---el London Aquatics Centre para los Juegos Olímpicos, el Museo Guggenheim de Taiwan, el Guangzhou Opera House en China. Pero, en realidad, la obsesión con el entorno, con el paisaje, con la topografía son elementos notables en sus diseños.

La relación que tienen sus proyectos con los sitios donde se implantan no es mimética, sino transformadora. Sus formas no son al azar; como por ejemplo, en el museo diseñado en Glasgow, en el cual la planta en forma de "S" se deriva de los techos de diente de sierra de las naves industriales que solían atracar allí. No hay memoria cultural en una forma futurista; eso se va adquiriendo con el tiempo (Giovanni, 2011).

\section{REFERENCIAS BIBLIOGRÁFICAS}

Barbosky, M. (2001). Arquitectura siglo XX. Milán: Editorial electa.

Barreneche, R.A. (2003). Zaha Hadid prepares for mainstream succes. Architectural Record, 191 (1), 74.

Elmhirst, S. (2011). Zaha Hadid's learning curve. New Statesman, 140 (5078), 14.

Evers, B. (2006). Teoría de la arquitectura: desde el renacimiento hasta la actualidad. Berlin: Editorial Taschen.

Forsyth, A. (2006). In Praise of Zaha. Journal of Architectural Education, 60 (2), 63-65. doi: 10.1111/j.1531$314 X .2006 .00082 . x$

Giovannini, j. (2011). Poetry in motion. Interior Design, 82 (10), $136-146$

Hadid, Z. (2006). Otro tipo de espacio. Una entrevista con Zaha Hadid. Detail- Revista de Arquitectura.

Hadid, Z. (2005) Obras Completas de Zaha Hadid. Ediciones Akal. Hadid, Z. (2005). Revista Axxis, vol. 148.

Jencks, Ch. (2004). El Nuevo paradigma de la arquitectura contemporánea. Valencia: Ediciones Generales de la Construcción.

Jodidio, Ph. (2001). New Forms. La arquitectura de los noventa. Milán: Editorial Taschen.

Mostafavi, M. (2001). El paisaje como planta (una conversación con Zaha Hadid). Madrid: Editorial el croquis.

Pontius, N.L. (2010). Cultural Center in Jordan influenced by Ancient City of Petra. Civil Engineering (08857024), 80 (5), 34-37.

Póns, J. (2008). Un código visual y arquitectónico visual. (Spanish). Revista De EGA, 13, 160 -169.
Spencer, D. (2010). Replicant urbanism: The architecture of Hadid's Central Building at BMW. Journal of Architecture, 15(2), 181-207. doi: 10.1080=13062361003791044

Wells, M. (2005) Rascacielos: Las torres del siglo XXI. Editorial H. Kliczclowski.

\section{FORMA DE CITAR ESTE ARTÍCULO}

Cabas García, Mauricio (2013). Zaha Hadid: fluidez de movimiento. Revista Arte y Diseño Facultad de Arquitectura, Arte y Diseño, Universidad Autónoma del Caribe, Barranquilla. ISSN 1692- 8555 Vol. 11 (N'.2) PP. $15-21$ 